\title{
Atypical carcinoma detected after regression of a "benign" oral white lesion. A case report
}

\author{
Jan Srubara ${ }^{\mathrm{a}}$ Tereza Uhrikova ${ }^{\mathrm{a}}$, Patricie Delongova ${ }^{\mathrm{b}}$
}

Background. Unlike leukoplakia, the smokers' lesion - a type of oral white lesion spontaneously regressing following cessation of smoking - is generally considered a non-serious condition and there is no recommendation for subsequent follow-up of such patients.

Case report. Here, however, we present the case of a patient (female, 56, smoker) in whom we detected a smoker's lesion which regressed completely, without any signs of abnormality or malignity. The only shadow of a doubt that led us to inviting her for another examination was an additional examination using a VELscope ${ }^{\circledR}$ autofluorescence device that revealed autofluorescence suppression on the site of the former lesion. Another examination one month later revealed a patch of epithelium with a normal color but a negligibly different light reflection in the center of the former lesion; VELscope ${ }^{\circledast}$ indicated the questionable area to still be suspect. Following excision using margins indicated by VELscope ${ }^{\oplus}(+2-3 \mathrm{~mm})$, histopathology revealed a squamous cell carcinoma. The secondary excision was carcinoma free and even after 5 years, the carcinoma has not recurred.

Conclusion. We would like to point out that apparent spontaneous regressions of oral white lesions following smoking cessation still need attention and close follow-up to make sure that a developing squamous cell carcinoma cannot pass undetected.

Key words: smokers' lesion, oral white lesion, squamous cell carcinoma, leukoplakia, regression, cessation of smoking

Received: October 18, 2019; Revised: December 1, 2019; Accepted: January 20, 2020; Available online: February 25, 2020 https://doi.org/10.5507/bp.2020.002

(c) 2021 The Authors; https://creativecommons.org/licenses/by/4.0/

aDepartment of Oral and Maxillofacial Surgery, University Hospital Ostrava, 17. listopadu 1790, 708 52, Ostrava, Czech Republic ${ }^{b}$ Department of Pathology, University Hospital Ostrava, 17. listopadu 1790, 708 52, Ostrava, Czech Republic Corresponding author: Jan Srubar, e-mail:jansrubar@seznam.cz

\section{INTRODUCTION}

In the past, all oral white lesions used to be summarily termed as "leukoplakia". With the introduction of the latest WHO terminology, however, the definition of leukoplakia was changed to "a white plaque of questionable risk having excluded (other) known diseases or disorders that carry no increased risk for cancer" . According to this nomenclature, it is suggested that if an oral white lesion disappears relatively rapidly, i.e., within 4-8 weeks after removing the etiological factor, the lesion should retrospectively be reclassified accordingly; for example, where smoking is the suspected etiological factor and the oral white lesion disappears within 8 weeks after smoking cessation, it should be reclassified to "smokers' lesion" and is generally considered to be of negligible risk to the patient ${ }^{2-4}$.

\section{CASE REPORT}

A patient (female, 56) came to our department with a well-delineated (approx. 1 x $1.5 \mathrm{~cm}$ ) area of white plaque that could not be removed by brushing. The lesion was located on the left underside of the tongue. No clinical signs of malignity were present, no palpable resistance was detected upon investigation. The patient had a long smoking history (approx. 10 cigarettes a day), suffered from no other known pathologies or diseases apart from an allergy to penicillin-type antibiotics, and had no history of surgical intervention in the oral cavity. According to the C-factor principle ${ }^{2}$, smoking cessation was recommended (which the patient complied with) and the patient was invited for a new appointment 4 weeks later.

After that period, the white lesion had regressed considerably and after an additional 4 weeks, no white plaque was found at the location of the lesion and no clinically observable marks of malignity or any other disorder were present. The only shadow of doubt was cast when performing an additional examination using VELscope ${ }^{\circledR}$ a device using autofluorescence for detecting the presence of potential dysplasia in the oral cavity (in areas with dysplasia, the normal autofluorescence is suppressed). However, autofluorescence examination is not considered to be sufficient proof of dysplasia and even a detailed investigation of the site did not reveal any other abnormality; therefore, according to the current nomenclature, the lesion was diagnosed as a suspected smoking-associated homogenous oral white lesion, currently in regression following elimination of the causal factor. The patient was, 
however, invited for another follow-up a month later. During that visit, examination revealed that the suppression of autofluorescence on the site of the former white lesion persisted (see below) and upon close examination of the site, a subtle difference in the appearance of the tissue on the site (approx. $5 \times 10 \mathrm{~mm}$ ) from the surrounding mucosa was noted, which was only apparent as a minuscule difference in the light reflection - the color was normal (Fig. 1A). The lesion was not causing any discomfort to the patient, even when pressure was applied, and no palpable resistance was detected. The patient was examined by three experienced consultants but the opinions were inconclusive. Regarding the VELscope ${ }^{\circledR}$ examination, the loss of fluorescence in the respective area exceeded the area that was suspect under the naked eye by a few millimeters while other tissues of the oral cavity appeared normal (Fig. 1B). In the end, in view of the above, as well as of the fact that the suspect tissue was on the site of a previous white lesion and the lesion was relatively small and distant from any important anatomical features, and that its removal was therefore unlikely to cause any major problems to the patient, a decision was made to surgically remove the suspect tissue.

Under local anesthesia, the border of the lesion was delineated using ink and compared to borders determined by VELscope ${ }^{\circledR}$ examination. Excision margins were determined to be 2-3 mm outside the VELscope ${ }^{\circledR}$-detected area. The lesion was subsequently excised and the wound was left to heal by secondary intention. The healing progressed without any complications other than a sensation of numbness of the tongue.

The histological examination of the excised lesion revealed a body covered with squamous cell epithelium evolving into a p53-positive, microinvasive squamous cell carcinoma with a proliferative activity higher than $15 \%$ and a lichenoid inflammatory infiltrate present in the stroma. The excision margins were clear of tumorous cells (Fig. 2).

The final diagnosis was squamous epithelial carcinoma of the left underside of the tongue, classification T1N0M0. A follow-up ultrasonography examination of the neck was negative. Following the final diagnosis, a second surgery was performed extending the original margins to the recommended margins for oral carcinoma, i.e., $20 \mathrm{~mm}$, and reconstructing the shape of the tongue. Histological examination of the removed tissue revealed fibrosis without any marks of carcinoma. The patient was subsequently followed up and even after 5 years, no recurrence has been observed. The patient's only complaint is that of reduced sensitivity of the tongue.

\section{DISCUSSION}

It is a well-known fact that smoking cessation can result in spontaneous regression of oral white lesions ${ }^{5,6}$. If the disappearance of the lesion is relatively quick, within 4-8 weeks, the final diagnosis should be in retrospect changed from "leukoplakia" to "smokers' lesion" ", No further recommendations related to the treatment or follow-up of the smokers' lesions was however found in the current literature. The sentiment is obvious - the causative agent was determined, the precancerous lesion disappeared, the patient is cured.

In our report, however, we present a case where despite a spontaneous regression of such a lesion, the underlying tissue developed a squamous epithelial carcinoma. It is even rarer considering the results of a study by Holmstrup et al. ${ }^{1}$ focused on the long-term outcome of potentially malignant lesions of the oral mucosa including leukoplakia. Their results showed that the only predictive factors associated with developing a carcinoma were homogeneity and, the size of the lesion, both of which indicated a low risk in this particular patient (the lesion was originally smaller than $2 \mathrm{~cm}$ and homogenous).

Making a clinical decision in such a case can be difficult. The tissue looked almost normal and had it not been for the use of the VELscope ${ }^{\circledR}$ device during the third visit, which cast a shadow of doubt on the otherwise clear diagnosis of a smoker's lesion and led us to perform another thorough investigation of the site due to the previous presence of leukoplakia a month later, the carcinoma development would have progressed much further before its detection. Indeed, even during the fourth visit, the very

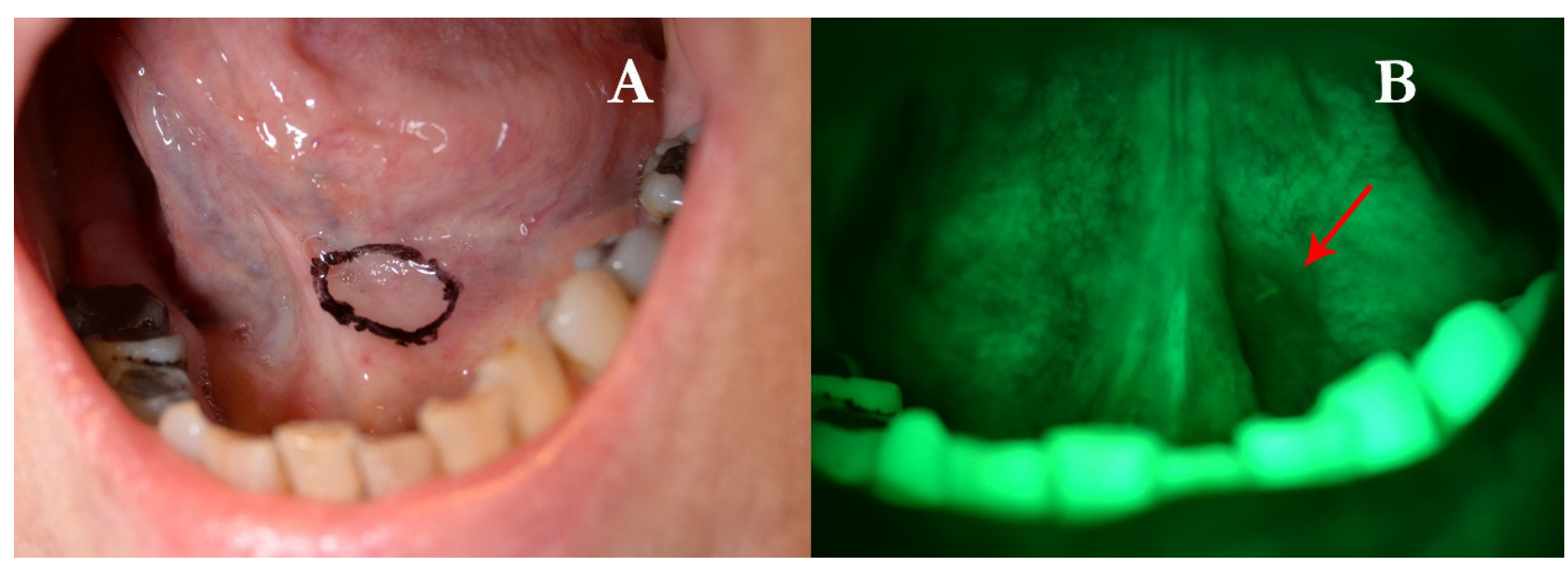

Fig. 1. The appearance of the lesion under normal light (A) and VELscope ${ }^{\circledR}(B)$. 


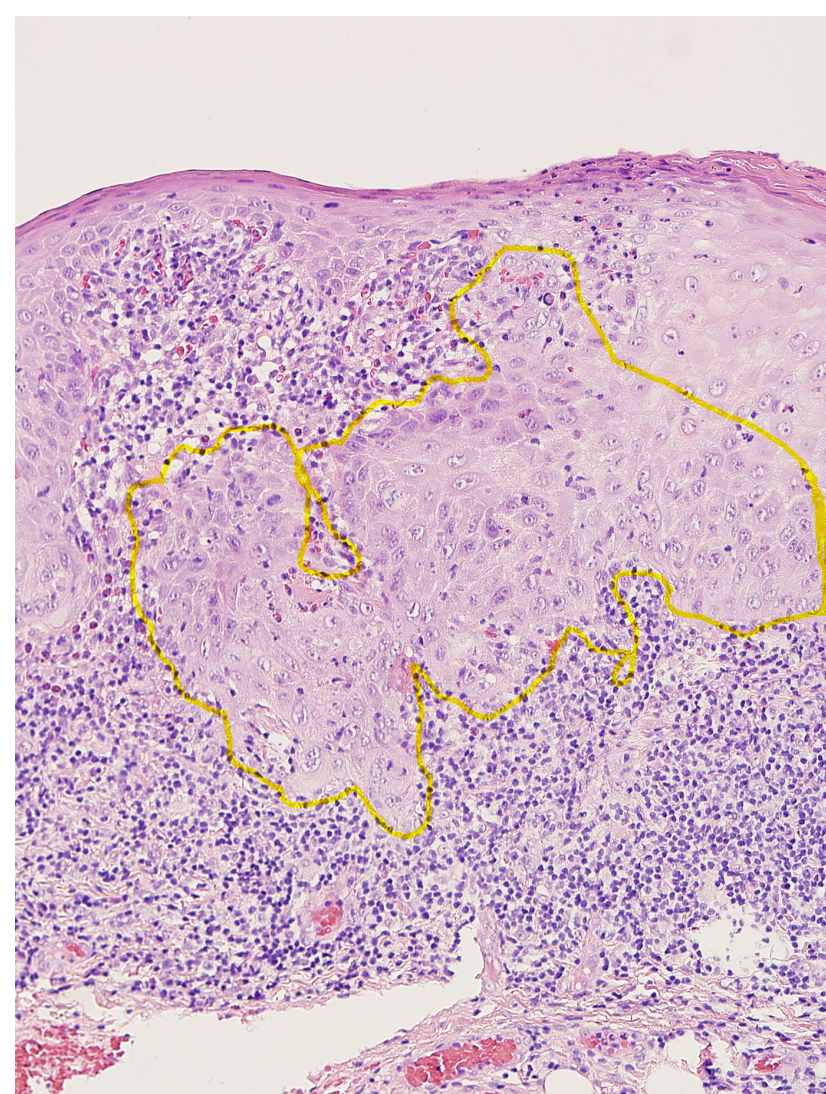

Fig. 2. Photo of histopathological examination, borders of carcinoma delineated in yellow (HE 10x).

slight abnormality could have been easily missed. Without any auxiliary evidence, the sensible decision would be to wait for several more weeks to find out if the tissue would normalize completely.

As mentioned above, VELscope ${ }^{\circledR}$ is an instrument based on the difference between autofluorescence of the normal and dysplastic cells - while fluorochromes of healthy cells react to excitation by emitting light of a specific wavelength that can be visualized using a proper filtration, the distribution of the fluorochromes is disrupted in dysplastic cells resulting in their dark appearance during the investigation. Recent reviews by Nagi et al. ${ }^{8}$ and Tiwari et al. ${ }^{9}$ reveal the controversy in using VELscope $\AA$, with reported results varying from "not worth doing", through "a valuable adjunctive tool" to "an excellent screening tool". The latest study by Shi et al. reported an excellent capability of VELscope ${ }^{\circledR}$ to identify high risk lesions (moderate/severe dysplasia/oral carcinoma) from low risk lesions on a cohort of 517 patients ${ }^{10}$. Autofluorescence imaging was also shown to be helpful in defining excision margins where the decision to excise is made ${ }^{11}$, which is in line with the reported case where a full excision was performed during the first VELscope ${ }^{\circledR}$-guided biopsy - this proved sufficient, and the secondary surgery aiming at increasing the excision margins did not reveal any signs of carcinoma or dysplasia. In general, the experience with VELscope $\AA$ at our clinic is probably most consistent with the "valuable adjunctive tool" conclusion, which also played a crucial role in the decision to excise the almost invisible lesion. Additional factors playing a role in the decision to excise the suspected lesion were the previous presence of a white lesion on the site and the relatively small size of the lesion not directly affecting any important anatomical structures and therefore a relatively low risk of complications.

We are well aware that we cannot draw any firm conclusion from a single case. Nevertheless, we still feel that it is justified to point out that even though some oral white lesions may disappear spontaneously after smoking cessation (which would lead to their reclassification as a mere successfully cured smokers' lesion), the patients should still be followed up for a prolonged period of time to ensure early detection of any possible malignancy developing on the site. Secondly, even negligible changes in the appearance of the tissue (no color changes, no clear definition of borders under the visible light, no palpable resistance, only a slight change in light reflection) may represent a developing carcinoma. Lastly, although the use of VELscope ${ }^{\circledR}$ and other autofluorescence imaging techniques is still a subject of much controversy, we believe that when appropriately used, it could be a valuable adjunctive tool when making a decision about biopsy, especially where the autofluorescence quenching persists even after the apparent regression of the white lesion. Furthermore, the excision margins determined using VELscope ${ }^{\circledR}$ were sufficient for full removal of the carcinoma, which was another benefit of its use in our case.

Acknowledgement: This work was supported by $\mathrm{MH} \mathrm{CZ}$ - DRO - FNOs/2013.

Authors contributions: JS: care for the patient, manuscript writing; TU: care for the patient, manuscript writing; PD: histopathological examination, manuscript writing.

Conflict of interest statement: The authors declare that they have no conflict of interest with regard to this work.

\section{REFERENCES}

1. Holmstrup P, Vedtofte P, Reibel J, Stoltze K. Long-Term Treatment Outcome of Oral Premalignant Lesions. Oral Oncol 2006;42(5):46174.

2. van der Waal I. Oral Leukoplakia, the Ongoing Discussion on Definition and Terminology. Med Oral Patol Oral Cir Bucal 2015;20(6):e685-92.

3. Carrard VC, van der Waal I. A Clinical Diagnosis of Oral Leukoplakia; a Guide for Dentists. Med Oral Patol Oral Cir Bucal 2018;23(1):e59-e64.

4. van der Waal I. Historical Perspective and Nomenclature of Potentially Malignant or Potentially Premalignant Oral Epithelial Lesions with Emphasis on Leukoplakia-Some Suggestions for Modifications. Oral Surg Oral Med Oral Pathol Oral Radiol 2018;125(6):577-81.

5. Banoczy J, Gintner Z, Dombi C. Tobacco Use and Oral Leukoplakia. J Dent Educ 2001;65(4):322-7.

6. Warnakulasuriya S, Dietrich T, Bornstein MM, Casals Peidro E, Preshaw PM, Walter C, Wennstrom JL, Bergstrom J. Oral Health Risks of Tobacco Use and Effects of Cessation. Int Dent J 2010;60(1):7-30.

7. Brouns ER, Baart JA, Bloemena E, Karagozoglu H, van der Waal I. The Relevance of Uniform Reporting in Oral Leukoplakia: Definition, Certainty Factor and Staging Based on Experience with 275 Patients. Med Oral Patol Oral Cir Bucal 2013;18(1):e19-26.

8. Nagi R, Reddy-Kantharaj YB, Rakesh N, Janardhan-Reddy S, Sahu S. Efficacy of Light Based Detection Systems for Early Detection of Oral Cancer and Oral Potentially Malignant Disorders: Systematic Review. Med Oral Patol Oral Cir Bucal 2016;21(4):e447-55. 
9. Tiwari L, Kujan O, Farah CS. Optical Fluorescence Imaging in Oral Cancer and Potentially Malignant Disorders: A Systematic Review. Oral Dis 2019; Feb 27. doi: 10.1111/odi.13071 [Epub ahead of print]

10. Shi L, Li C, Shen X, Zhou Z, Liu W, Tang G. Potential Role of Autofluorescence Imaging in Determining Biopsy of Oral Potentially
Malignant Disorders: A Large Prospective Diagnostic Study. Oral Oncol 2019;98:176-9. doi: 10.1016/j.oraloncology.2019.08.006 11. Farah CS, Kordbacheh F, John K, Bennett N, Fox SA. Molecular Classification of Autofluorescence Excision Margins in Oral Potentially Malignant Disorders. Oral Dis 2018;24(5):732-40. 